\title{
Influência do tipo de transdutor na deficiência auditiva de grau profundo $* * * *$
}

\author{
Influence of the type of transducer in profound hearing loss
}

\author{
Andréa Tortosa Marangoni* \\ Daniela Gil**
}

*Fonoaudióloga. Especializanda em Audiologia pela Universidade Federal de São Paulo. Endereço para correspondência: Rua Nicarágua, 140 São Paulo - SP - CEP 12030-500 (andreatortosa@hotmail.com).

**Fonoaudióloga. Doutora em Distúrbios da Comunicação Humana pela Universidade Federal de São Paulo. Professor Adjunto do Departamento de Fonoaudiologia da Universidade Federal de São Paulo.

****Trabalho Realizado nos Ambulatórios de Audiologia Clínica do Departamento de Fonoaudiologia da Universidade Federal de São Paulo.

Artigo Original de Pesquisa

Artigo Submetido a Avaliação por Pares

Conflito de Interesse: não

Recebido em 12.11.2008.

Revisado em 26.02.2009; 07.08.2009.

Aceito para Publicação em 28.08.2009.

\begin{abstract}
Background: individuals with profound hearing loss may present vibrotactile responses when tested with supra-aural earphones due to the large skull area exposed to vibration in these transducers. Aim: to verify the influence of the type of transducer when assessing air conducted pure tone thresholds in individuals with profound sensorineural hearing loss. Method: 50 individuals, ranging in age from 16 to 55 years, were submitted to a clinical history questionnaire, meatoscopy, and pure tone and speech audiometry, using both TDH-39 supra-aural earphones and ER-3A insert earphones. Results: in both pure tone and speech testing, thresholds were higher when obtained using insert earphones, with statistical significant differences for $250 \mathrm{~Hz}$ and $1000 \mathrm{~Hz}$. Considering right and left ear testing with the different transducers, this difference was greater for the right ear, with statistical significant differences only for $250 \mathrm{~Hz}$. Regarding gender, it was observed that the difference between the transducers was greater in males, with statistical significant differences for $250 \mathrm{~Hz}$. However, in the SDT (Speech Detection Threshold), the greatest difference was found in females. Conclusion: people with bilateral sensorineural profound hearing loss presented higher (worse) thresholds with insert earphones (ER-3A) than with supra aural earphones (TDH-39) for low frequencies $(250 \mathrm{~Hz}$ and $1000 \mathrm{~Hz})$, confirming the existence of vibrotactile responses with supra aural earphones.
\end{abstract}

Key Words: Earphones; Vibrotactile; Pure Tone Audiometry; Speech Audiometry..

\section{Resumo}

Tema: indivíduos com deficiência auditiva de grau profundo podem apresentar respostas por vibração com os fones supra-aurais devido a grande área do crânio exposta à vibração nestes transdutores. Objetivo: verificar a influência do tipo de transdutor na obtenção dos limiares auditivos por via aérea em indivíduos com deficiência auditiva neurossensorial de grau profundo. Método: 50 indivíduos, com idades variando de 16 a 55 anos, foram submetidos a anamnese, meatoscopia, e posteriormente, a audiometria tonal liminar e limiar de deteç̧ão de voz (LDV). As audiometrias tonal e vocal foram realizadas tanto com fones supraaurais TDH-39 quanto com os fones de inserção ER-3A. Resultados: nas audiometrias tonal e vocal, notamos que, com o fone de inserção, foram obtidos limiares auditivos mais elevados do que os obtidos com o fone supra-aural, com significância estatística nas frequiências de $250 \mathrm{~Hz}$ e $1000 \mathrm{~Hz}$. Ao observarmos a diferença entre os resultados obtidos com o transdutor TDH-39 e o ER-3A em cada orelha separadamente, notamos que a diferença foi maior na orelha direita com significância estatística somente na freqüência de $250 \mathrm{~Hz}$. Em relação ao sexo, notamos que as diferenças entre os transdutores foram maiores no sexo masculino com significância estatística na frequiência de $250 \mathrm{~Hz}$. No entanto, no LDV, a diferença maior foi encontrada no sexo feminino. Conclusão: deficientes auditivos neurossensorias de grau profundo bilateral apresentam limiares de audibilidade mais elevados (piores) com os fones de inserção do que com os fones supra-aurais nas frequiências baixas, evidenciando a existência das respostas por vibração com os fones supra-aurais.

Palavras-Chave: Fones de Ouvido; Vibração; Audiometria de Tons Puros; Audiometria de Fala. 


\section{Introdução}

Existem três tipos de fones na prática da audiologia clínica (supra-aurais, circum-aurais e de inserção) que são utilizados para obtenção de limiares de audibilidade tanto para tons puros como para estímulos de fala ${ }^{1}$.

Nos fones supra-aurais, transdutores convencionais em audiologia clínica, o coxim do fone é pressionado contra o pavilhão auricular. Devido à grande área do crânio exposta à vibração nestes transdutores, há possibilidade de deficientes auditivos neurossensoriais de grau profundo apresentarem respostas por vibração, impossibilitando a medida exata da sensibilidade auditiva nesses pacientes, especialmente nas freqüências baixas ${ }^{2}$.

Os fones de inserção ER-3A foram desenvolvidos para reproduzir as características eletroacústicas dos fones TDH-39. No entanto, o fabricante recomendou o uso de fatores de correção quando esses transdutores são utilizados em equipamentos calibrados para os fones supra-aurais $^{3}$. A área de contato entre o fone de inserção e a pele é mínima, reduzindo a área da cabeça exposta ao estímulo sonoro devido a sua adaptação dentro do meato acústico externo (MAE) por meio de plugs de espuma descartáveis e desta forma, ocorre a redução das respostas por vibração.

Diversos estudos concluíram que, além de deixarem o exame mais confortável, os fones de inserção, quando comparados aos supra aurais, possibilítam maior confiabilidade ao exame; maior atenuação do ruído ambiental; maior atenuação interaural, podendo reduzir ou até eliminar a necessidade de mascaramento contralateral; diminuição do efeito de oclusão na testagem da via óssea e redução significante do risco de colabamento do $\mathrm{MAE}^{4-7}$. No entanto, alguns autores citaram como desvantagens dos fones de inserção a variabilidade da geometria do meato acústico externo ${ }^{1,8-10}$, faixa dinâmica insuficiente nas freqüências altas e problemas relacionados com a higiene na utilização dos plugs de espuma ${ }^{1}$.

Frente ao exposto, o objetivo deste estudo é verificar a influência do tipo de transdutor na obtenção dos limiares auditivos por via aérea de indivíduos com deficiência auditiva neurossensorial de grau profundo, comparando as respostas obtidas com os fones supraaurais TDH-39 e de inserção ER-3A.

\section{Método}

O projeto desta pesquisa recebeu parecer favorável do Comitê de Ética em Pesquisa da
Unifesp sob o número 1044/07. Todos os sujeitos antes da avaliação foram informados sobre os procedimentos a serem realizados e assinaram um termo de consentimento livre e esclarecido autorizando sua participação no estudo conforme resolução 196/96.

A população dessa pesquisa foi selecionada e avaliada nos Ambulatórios de Audiologia Clínica do Departamento de Fonoaudiologia da Universidade Federal de São Paulo. Foram avaliados indivíduos encaminhados para avaliação audiológica para obtenção do benefício do transporte coletivo gratuito e os critérios de elegibilidade para inclusão na amostra foram: presença de deficiência auditiva neurossensorial bilateral de grau profundo simétrica com restos auditivos até no máximo $1000 \mathrm{~Hz}$, curva timpanométrica tipo $A$ e ausência de comprometimentos neurológico e/ou cognitivo evidentes.

Todos os indivíduos foram submetidos a uma anamnese e em seguida, à meatoscopia.

Foram avaliados 150 indivíduos e destes, foram selecionados 50, pois o restante não contemplou todos os critérios de elegibilidade propostos. Dos 50 indivíduos, 25 eram do sexo feminino e 25 do sexo masculino com idades variando entre 17 e 55 anos (média $=35,52)$ e 16 e 55 anos (média $=33,56$ ) respectivamente.

$\mathrm{Na}$ anamnese pudemos observar que $82 \%$ dos indivíduos apresentaram perda auditiva congênita e $18 \%$ perda auditiva adquirida. Em relação à presença de zumbido e tontura, $6 \%$ e $24 \%$ da amostra apresentaram os sintomas, respectivamente.

Os sujeitos foram submetidos à avaliação audiológica composta por audiometria tonal limiar (via aérea e via óssea) e logoaudiometria (LDV limiar de detecção de voz).

A avaliação audiológica (audiometria tonal limiar e logoaudiometria) foi realizada em cabina acústica, com o audiômetro de marca MA-41 Interacoustics calibrado segundo o padrão ANSI 1969. A audiometria tonal limiar e a audiometria vocal foram realizadas com fones supra-aurais TDH-39, e posteriormente, com os fones de inserção ER-3A. Para a utilização dos fones ER-3A no mesmo equipamento, foram utilizados os fatores de correção, sugeridos pelo fabricante, a serem acrescentados nos limiares obtidos (Etymotic Research, 1985), a saber: $250 \mathrm{~Hz}=5 \mathrm{~dB} ; 500 \mathrm{~Hz}=0 \mathrm{~dB}$; $1000 \mathrm{~Hz}=5 \mathrm{~dB} ; 2000 \mathrm{~Hz}=5 \mathrm{~dB} ; 3000 \mathrm{~Hz}=5 \mathrm{~dB} ; 4000 \mathrm{~Hz}$ $=0 \mathrm{~dB} ; 6000 \mathrm{~Hz}=-10 \mathrm{~dB} ; 8000 \mathrm{~Hz}=-10 \mathrm{~dB}$.

De acordo com as recomendações do fabricante, para que o fone de inserção tenha o desempenho 
ideal, a inserção do plug deve ser profunda (2-3mm), garantindo uma posição total de inserção de mais ou menos $16 \mathrm{~mm}$ de profundidade no meato acústico externo medida a partir do plano da concha ${ }^{3,11-12}$.

Durante a pesquisa dos limiares de audibilidade, realizada nas freqüências de 250 a $8000 \mathrm{~Hz}$, os indivíduos foram instruídos a levantar a mão para todos os estímulos ouvidos, mesmo que em fraca intensidade. A freqüência inicial do teste foi de 1000 $\mathrm{Hz}$ e posteriormente foram obtidos os limiares de audibilidade nas freqüências de 2000, 3000, 4000, $6000,8000,500$ e $250 \mathrm{~Hz}$, nesta ordem.

A técnica utilizada na obtenção dos limiares com ambos os transdutores foi o método descendente, a qual envolve os seguintes passos: a) o estímulo sonoro foi apresentado em um nível de intensidade facilmente percebido pelo examinando; b) a intensidade foi diminuída em intervalos de $10 \mathrm{~dB}$ até que o estímulo se tornasse inaudível; c) a intensidade foi aumentada em intervalos de $5 \mathrm{~dB}$ até que o indivíduo percebesse novamente a presença do som. Foi considerado o limiar de audibilidade o menor nível de intensidade no qual o paciente respondeu duas das quatro apresentações (50\% das vezes), após a realização dos passos citados acima.

$\mathrm{Na}$ audiometria vocal (Limiar de Detecção de Voz - LDV), os indivíduos foram instruídos a levantar a mão para todos os estímulos de fala percebidos (pa pa pa). O LDV foi realizado a viva voz inicialmente a $100 \mathrm{~dB}$ NA, e foi registrado o menor nível de intensidade no qual foi detectado o som.

Para a análise estatística, utilizamos os testes não paramétricos de Wilcoxon e de Mann-Whitney. Para fins estatísticos, quando os indivíduos apresentaram ausência de respostas, consideramos um valor de 10 decibéis acima da saída máxima do aparelho. Os resultados com significância estatística foram destacados com asterisco (*) e com tendência à significância foram destacados com o símbolo sustenido (\#). Os intervalos de confiança foram construídos com $95 \%$ de confiança estatística e foi adotado nível de significância de 5\% $(0,05)$.

\section{Resultados}

Nas Tabelas de 1 a 3 encontram-se descritos os resultados obtidos com os fones supra-aurais TDH39 e de inserção ER-3A e suas comparações segundo as variáveis: sexo e lado de orelha.

TABELA 1. Valores descritivos (média, mediana e desvio padrão) dos limiares de audibilidade obtidos com os fones supra-aurais TDH-39 e de inserção ER-3A em 50 indivíduos de ambos os sexos.

\begin{tabular}{|c|c|c|c|c|c|}
\hline \multicolumn{2}{|c|}{ Transdutores } & Média & Mediana & Desvio Padrão & P-Valor \\
\hline \multirow[t]{2}{*}{$250 \mathrm{~Hz}$} & ER-3A & 88,8 & 90 & 12,85 & $0,025^{*}$ \\
\hline & TDH-39 & 85,0 & 85 & 11,07 & \\
\hline \multirow[t]{2}{*}{$500 \mathrm{~Hz}$} & ER-3A & 96,7 & 95 & 11,50 & 0,483 \\
\hline & TDH-39 & 95,3 & 95 & 9,89 & \\
\hline \multirow[t]{2}{*}{$1 \mathrm{kHz}$} & ER-3A & 109,1 & 110 & 8,12 & $0,014 *$ \\
\hline & TDH-39 & 106,1 & 105 & 8,66 & \\
\hline \multirow[t]{2}{*}{$2 \mathrm{kHz}$} & ER-3A & 114,9 & 115 & 9,10 & 0,751 \\
\hline & TDH-39 & 115,4 & 115 & 9,99 & \\
\hline \multirow[t]{2}{*}{$3 \mathrm{kHz}$} & ER-3A & 121,7 & 120 & 10,52 & 0,124 \\
\hline & TDH-39 & 119,5 & 120 & 11,58 & \\
\hline \multirow[t]{2}{*}{$4 \mathrm{kHz}$} & ER-3A & 122,4 & 130 & 12,86 & 0,562 \\
\hline & TDH-39 & 123,4 & 130 & 11,48 & \\
\hline \multirow[t]{2}{*}{$6 \mathrm{kHz}$} & ER-3A & 117,5 & 120 & 8,72 & 0,690 \\
\hline & TDH-39 & 117,5 & 120 & 8,45 & \\
\hline \multirow[t]{2}{*}{$8 \mathrm{kHz}$} & ER-3A & 108,9 & 110 & 5,84 & 0,235 \\
\hline & TDH-39 & 107,8 & 110 & 8,02 & \\
\hline \multirow[t]{2}{*}{ LDV } & ER-3A & 85,9 & 85 & 12,03 & 0,507 \\
\hline & TDH-39 & 86,7 & 85 & 9,46 & \\
\hline
\end{tabular}

Legenda: $\mathrm{kHz}=$ quilo hertz; $\mathrm{p}<0,05$ Wilcoxon; LDV = limiar de detecção de voz. 
TABELA 2. Valores descritivos (média, mediana e desvio padrão) da diferença dos limiares de audibilidade obtidos com os fones supra-aurais TDH-39 de inserção ER-3A nas orelhas esquerda e direita em 50 indivíduos de ambos os sexos.

\begin{tabular}{|c|c|c|c|c|c|}
\hline & Diferença & Média & Mediana & Desvio Padrão & P-Valor \\
\hline \multirow[t]{2}{*}{$250 \mathrm{~Hz}$} & orelha direita & $-5,60$ & $-5,0$ & 8,37 & \multirow{2}{*}{$<0,001 *$} \\
\hline & orelha esquerda & $-2,10$ & 0,0 & 8,58 & \\
\hline \multirow[t]{2}{*}{$500 \mathrm{~Hz}$} & orelha direita & $-1,90$ & 0,0 & 5,52 & \multirow{2}{*}{0,336} \\
\hline & orelha esquerda & $-0,80$ & 0,0 & 6,17 & \\
\hline \multirow[t]{2}{*}{$1 \mathrm{kHz}$} & orelha direita & $-2,90$ & $-5,0$ & 4,53 & \multirow{2}{*}{0,672} \\
\hline & orelha esquerda & $-3,00$ & $-5,0$ & 4,74 & \\
\hline \multirow[t]{2}{*}{$2 \mathrm{kHz}$} & orelha direita & 0,30 & 0,0 & 6,26 & \multirow{2}{*}{0,754} \\
\hline & orelha esquerda & 0,70 & 0,0 & 5,98 & \\
\hline \multirow[t]{2}{*}{$3 \mathrm{kHz}$} & orelha direita & $-2,10$ & 0,0 & 6,32 & \multirow{2}{*}{0,665} \\
\hline & orelha esquerda & $-2,20$ & 0,0 & 6,16 & \\
\hline \multirow[t]{2}{*}{$4 \mathrm{kHz}$} & orelha direita & 1,30 & 0,0 & 4,93 & \multirow{2}{*}{0,480} \\
\hline & orelha esquerda & 0,80 & 0,0 & 5,28 & \\
\hline \multirow[t]{2}{*}{$6 \mathrm{kHz}$} & orelha direita & 0,50 & 0,0 & 2,72 & \multirow{2}{*}{$0,047^{*}$} \\
\hline & orelha esquerda & $-0,50$ & 0,0 & 3,54 & \\
\hline \multirow[t]{2}{*}{$8 \mathrm{kHz}$} & orelha direita & $-1,10$ & 0,0 & 4,55 & \multirow{2}{*}{1,000} \\
\hline & orelha esquerda & $-1,10$ & 0,0 & 4,08 & \\
\hline \multirow[t]{2}{*}{ LDV } & orelha direita & 0,40 & 0,0 & 6,38 & \multirow{2}{*}{0,257} \\
\hline & orelha esquerda & 1,20 & 2,5 & 7,18 & \\
\hline
\end{tabular}

Legenda: $\mathrm{kHz}=$ quilo hertz; $\mathrm{p}<0,05$ Wilcoxon; $\mathrm{LDV}=$ limiar de detecção de voz.

TABELA 3. Valores descritivos (média, mediana e desvio padrão) da diferença dos limiares de audibilidade obtidos com os fones supra-aurais TDH-39 de inserção ER-3A em 25 indivíduos de cada sexo (feminino e masculino).

\begin{tabular}{|c|c|c|c|c|c|}
\hline & nça & Média & Mediana & Desvio Padrão & P-Valor \\
\hline \multirow[t]{2}{*}{$250 \mathrm{~Hz}$} & feminino & $-1,50$ & 0,0 & 8,28 & \multirow{2}{*}{$0,004^{*}$} \\
\hline & masculino & $-6,20$ & $-5,0$ & 8,36 & \\
\hline \multirow[t]{2}{*}{$500 \mathrm{~Hz}$} & feminino & $-0,40$ & 0,0 & 5,52 & \multirow{2}{*}{0,150} \\
\hline & masculino & $-2,30$ & 0,0 & 6,08 & \\
\hline \multirow[t]{2}{*}{$1 \mathrm{kHz}$} & feminino & $-2,80$ & 0,0 & 4,76 & \multirow{2}{*}{0,444} \\
\hline & masculino & $-3,10$ & $-5,0$ & 4,51 & \\
\hline \multirow[t]{2}{*}{$2 \mathrm{kHz}$} & feminino & 1,00 & 0,0 & 5,71 & \multirow{2}{*}{0,428} \\
\hline & masculino & 0,00 & 0,0 & 6,47 & \\
\hline \multirow[t]{2}{*}{$3 \mathrm{kHz}$} & feminino & $-2,60$ & 0,0 & 6,08 & \multirow{2}{*}{0,399} \\
\hline & masculino & $-1,70$ & 0,0 & 6,36 & \\
\hline \multirow[t]{2}{*}{$4 \mathrm{kHz}$} & feminino & 1,10 & 0,0 & 5,47 & \multirow{2}{*}{0,810} \\
\hline & masculino & 1,00 & 0,0 & 4,74 & \\
\hline \multirow[t]{2}{*}{$6 \mathrm{kHz}$} & feminino & 0,00 & 0,0 & 1,75 & \multirow{2}{*}{0,500} \\
\hline & masculino & 0,00 & 0,0 & 4,16 & \\
\hline \multirow[t]{2}{*}{$8 \mathrm{kHz}$} & feminino & $-0,90$ & 0,0 & 4,00 & \multirow{2}{*}{0,477} \\
\hline & masculino & $-1,30$ & 0,0 & 4,61 & \\
\hline \multirow[t]{2}{*}{ LDV } & feminino & 3,00 & 5,0 & 6,47 & \multirow{2}{*}{$0,001 *$} \\
\hline & masculino & $-1,40$ & 0,0 & 6,39 & \\
\hline
\end{tabular}

Legenda: $\mathrm{kHz}=$ quilo hertz; $\mathrm{p}<0,05$ Mann-Whitney; LDV = limiar de detecção de voz. 


\section{Discussão}

$\mathrm{Na}$ Tabela 1, foram verificados os valores descritivos dos limiares de audibilidade obtidos com os fones supra-aurais TDH-39 e de inserção ER-3A em 50 indivíduos de ambos os sexos. Pode-se observar que, com o fone de inserção, foram obtidos limiares de audibilidade mais elevados nas freqüências $250 \mathrm{~Hz}, 500 \mathrm{~Hz}, 1000 \mathrm{~Hz}, 3000 \mathrm{~Hz}$ e $8000 \mathrm{~Hz}$ do que os obtidos com o fone supra-aural TDH-39, sendo estatisticamente significante nas freqüências de $250 \mathrm{~Hz}$ e $1000 \mathrm{~Hz}$. Estes resultados podem ser explicados pelo fato de deficientes auditivos neurossensoriais de grau profundo bilateral, poderem apresentar resposta por vibração com o fone supra-aural especialmente nas freqüências baixas $(250 \mathrm{~Hz}, 500 \mathrm{~Hz}$ e $1000 \mathrm{~Hz})$, devido a grande área do crânio exposta à vibração, dificultando a medida exata da sensibilidade auditiva nesses pacientes, o que não ocorre com os fones de inserção.

A presença de respostas vibrotáteis pode comprometer o encaminhamento adequado do caso e em algumas situações, pode explicar as diferenças de desempenho existentes com uso de amplificação em indivíduos com deficiência auditiva de grau profundo com restos auditivos em frequiências baixas, uma vez que indivíduos com respostas vibrotáteis podem apresentar menor benefício com o uso da amplificação.

Na literatura especializada, foi encontrado um estudo $^{2}$ que citou como uma das vantagens dos fones de inserção, a diminuição das respostas que são mais vibrotáteis do que auditivas, confirmando nossos achados.

Com relação às demais frequiências (Tabela 1), pode-se observar que os resultados ou foram iguais, ou foram semelhantes com os dois tipos de transdutores (diferença máxima de $1,7 \mathrm{~dB}$ ). Além disso, deve haver cautela ao considerar esses resultados, visto que poucos indivíduos da amostra apresentaram restos auditivos nas freqüências acima de $1000 \mathrm{~Hz}$. Sendo assim, esta semelhança entre os transdutores não deve ser generalizada para populações com outras características audiológicas.

Na Tabela 2, está demonstrada a diferença entre os resultados obtidos com o transdutor TDH-39 e o ER-3A em cada orelha separadamente. Esta diferença foi obtida ao subtrair a média dos resultados encontrados com os fones supra-aurais e de inserção em cada orelha. Nota-se que a diferença entre os transdutores foi maior na orelha direita nas freqüências de $250 \mathrm{~Hz}, 500 \mathrm{~Hz}, 4000 \mathrm{~Hz}$, sendo estatisticamente significante somente na freqüência de $250 \mathrm{~Hz}$. Nas demais freqüências, com exceção de $6000 \mathrm{~Hz}$ e $8000 \mathrm{~Hz}$, e no LDV, esta diferença foi maior na orelha esquerda, sem significância estatística. Deve-se observar que nas freqüências $2000 \mathrm{~Hz}, 4000 \mathrm{~Hz}, 6000 \mathrm{~Hz}$ (somente na orelha direita) e no LDV, a diferença foi positiva, ou seja, os limiares obtidos com o fone TDH-39 foram mais elevados dos que encontrados com o fone ER-3A.

$\mathrm{Na}$ Tabela 3, pode-se observar a diferença entre os resultados obtidos com os transdutores TDH39 e ER-3A em cada sexo separadamente. Nota-se que a diferença entre os transdutores foram maiores no sexo masculino nas freqüências de $250 \mathrm{~Hz}, 500 \mathrm{~Hz}$, $1000 \mathrm{~Hz}$, e $8000 \mathrm{~Hz}$, sendo estatisticamente significante somente na frequiência de $250 \mathrm{~Hz}$, a favor do TDH-39, ou seja, os limiares auditivos obtidos com o fone ER-3A foram mais elevados (piores) dos que os encontrados com o fone TDH-39. Nas demais freqüências e no LDV, esta diferença foi maior no sexo feminino, com significância estatística somente no LDV, a favor do ER-3A, ou seja, os limiares auditivos obtidos com o fone TDH-39 foram mais elevados dos que encontrados com o fone ER-3A.

Muitos estudos citaram as variáveis que interferem na obtenção dos limiares com os fones de inserção, dentre elas está as diferenças individuais na geometria do meato acústico externo $^{1,9-10}$.

A variabilidade da geometria do meato acústico externo é citada como uma das desvantagens dos fones de inserção por vários pesquisadores ${ }^{1,8-10}$.

Pensando nas diferenças existentes entre o tamanho e a geometria do meato acústico externo entre homens e mulheres, podemos esperar algumas diferenças nos limiares obtidos com os fones de inserção. Se pensarmos que o meato acústico externo das mulheres é menor e mais curto do que o dos homens, poderíamos inferir que o espaço residual no meato acústico após a inserção do plug de espuma é também menor nas mulheres, o que contribuiria para diminuir a quantidade de ruído fisiológico neste espaço e conseqüentemente possibilitaria a obtenção de limiares mais baixos ${ }^{9}$. Isto pode explicar o achado deste estudo, na qual encontramos uma piora significativa nos limiares com os fones de inserção quando comparados com os fones supra-aurais no sexo masculino, enquanto que no sexo feminino, os resultados entre os transdutores foram semelhantes ou melhores com os fones de inserção quando comparados com os fones supra-aurais. 
Com base nos resultados encontrados, pudemos comprovar a existência das respostas por vibração com os fones supra-aurais, demonstrando a utilidade dos fones de inserção na população com deficiência auditiva de grau profundo de modo a contribuir para um planejamento mais efetivo da reabilitação destes indivíduos.

\section{Conclusão}

A partir da análise dos resultados podemos concluir que deficientes auditivos neurossensoriais de grau profundo bilateral apresentam limiares de audibilidade mais elevados (piores) com os fones de inserção (ER-3A) do que com os fones supraaurais (TDH-39) nas frequiências baixas $(250 \mathrm{~Hz}$ e $1000 \mathrm{~Hz}$ ), sendo a diferença maior, comparando os transdutores, na orelha direita e no sexo masculino na freqüência de $250 \mathrm{~Hz}$.

\section{Referências Bibliográficas}

1. Zwislocki $\mathbf{J}$ et al. Earphones in audiometry. The Journal of the Acoustical Society of America. 1988;83(4):1688-9.

2. Killion MC \& Villchur E. Coments on "Earphones in Audiometry". J Acoust Soc Am. 1988;85(4):1755-78.

3. Gil D, Borges ACLC. Fones de inserçäo: um estudo em indivíduos audiologicamente normais. Rer. Bras. Otorrinolaringol. 2001;67:480-7.

4. Lilly DJ \& Purdy JK. On the routine use of tubephone insert earphones. Am J Audiol. 1993;2:17-20.

5. Clemis JD, Ballad WJ, Killion MC. Clinical use of an insert earphone. The Annals of Otology, Rhinology, and Laryngology. 1986;95:520-4.

6. Yantis PA. Avaliação dos limiares auditivos por via aérea. In: Katz J. Tratado de Audiologia Clínica. 4a ed. São Paulo: Manole; 1999. p. 97-108.

7. Ramos J. Atenuação interaural: estudo comparativo com dois tipos de transdutores [Trabalho de Conclusão de Curso]. São Paulo: Universidade Federal de São Paulo; 2006.
8. Cox RM, McDaniel MD. Reference equivalent threshold levels for pure tone and 13 octare noise bands: insert earphone and TDH 49 earphone. J Acoust Soc Am. 1986;79(2):443-6.

9. Larson VD, Cooper WA, Talbott RE, Schwartz DM, Ahlstrom C, DeChichis AR. Reference threshold sound pressure levels for the TDH-50 and ER-3A earphones. J Acoust Soc Am. 1988;84:46-51.

10. Valente M, Potts LG, Valente M, Vass W, Goebel J. Intersubject Variability of Real-Ear Sound Pressure Level: Conventional and Insert Earphones. J Am Acad Audiol. 1994;5:390-8.

11. Wilber LA, Kruger B, Killion MC. Reference thresholds for the ER-3A insert earphone. J Acoust Soc Am. 1988;83(2):669-76.

12. Clark JL, Roeser RJ. Three studies comparing performance of the ER-3A tubephone with the TDH-50P earphone. Ear and Hearing. Oct 1988;9(5):268-74. 